\title{
Detection of serum antibodies and circulating antigens in a chimpanzee experimentally infected with Onchocerca volvulus
}

\author{
Niklaus Weiss ${ }^{1}$, M. C. van Den Ende ${ }^{2}$, E. J. Albiez ${ }^{3}$, V. K. Barbiero ${ }^{4}$, Karen Forsyth ${ }^{5}$ \\ AND A. M. PRINCE \\ ${ }^{1}$ Laboratory of Immunoparasitology, Swiss Tropical Institute, Basel, Switzerland; ${ }^{2}$ The Lindsley F. Kimball \\ Research Institute of the New York Blood Center, New York, N.Y. 10021, USA; and Vilab II, The Liberian \\ Institute for Biomedical Research, Robertsfield, Liberia; ${ }^{3}$ Bernhard-Nocht Institute for Nautical and Tropical \\ Diseases, 2000 Hamburg 4, Federal German Republic; ${ }^{4}$ Division of Communicable Diseases, Agency for \\ International Development, Washington, D.C. 20523, USA; ${ }^{5}$ Immunoparasitology Unit, The Walter and Eliza \\ Hall Institute of Medical Research, Melbourne, Victoria 3050, Australia
}

\begin{abstract}
The course of the humoral immune response was followed in a chimpanzee experimentally infected over 27 weeks with a total of 168 Onchocerca volvulus 3rd-stage larvae obtained from naturally infected wild-caught blackflies. Antibodies against an adult worm extract could be detected by ELISA from week 16 onwards (after the inoculation of 44 larvae). Peak antibody levels were observed between weeks 66 and 74 (about one year after the last larval injection). Thereafter, antibody levels markedly decreased but rose again after week 120. First microfilariae could be detected from week 124 onwards. Microfilarial counts remained low (not more than two microfilariae per skin snip) until the end of the observation period. High levels of $\operatorname{IgM}$ antibodies against adult $O$. volvulus antigens were detectable between weeks 26 and 80 by ELISA. Total IgE levels were found to be only marginally elevated during the course of the infection. Circulating parasite antigens were only detectable for a short time (weeks 34 to 44) of the prepatent period by immuno-radiometric assays (IRMAs) using monoclonal antibodies which were raised against $O$. gibsoni eggs. Competitive radio-immuno-assays detected host antibodies inhibiting binding of ${ }^{125}$ I-labelled monoclonal antibodies to parasite antigens from week 28 onwards. Host antibodies clearly interfere later in infection with the detection of circulating antigens.
\end{abstract}

\section{Introduction}

DUKE (1962) has shown that Onchocerca volvulus can be transmitted experimentally to the chimpanzee. Long-term parasitological observations were reported from 32 chimpanzees inoculated with third stage larvae (DUKE, 1980). After a prepatent period of one to two years, the patent microfilarial infection lasted for a maximum of nine years after the last larval injection. In view of the difficulty in diagnosing early stages of onchocerciasis infections, recognized as a high priority by the Onchocerciasis Control Programme (OCP) in West Africa, the analysis of serum samples of experimentally infected chimpanzees is of great interest. The serial bleedings of a chimpanzee, infected in 1980, gave us the opportunity for a collaborative longitudinal study analysing the antibody responses to $O$. volvulus antigens and the presence of circulating parasite antigens with a recently developed immunoradiometric assay using monoclonas antibodies raised against $O$. gibsoni eggs (FORSYTH et al., 1984).

\section{Materials and Methods}

\section{Infections and parasitological examination}

A four-year-old female chimpanzee (no. 114) was subcutaneously injected at the hip and in the back with a total of 168 live third-stage larvae over a period of 27 weeks (April 22 to October 28, 1980) at irregular intervals (see Fig. 1). The larvae were collected from wild-caught blackflies. Methods for collection and dissection of blackflies have been described in detail (BARBIERO \& TRPIS, 1984). No blood microfilariae could be detected before the $O$. volvulus infection. Monthly skin snips using a "Walzer" scleral punch (calibre $2 \cdot 3 \mathrm{~mm}$; L. Klein, Heidelberg, FRG) at the right and left gluteal region and on the right and left back below the shoulder blade) were taken at monthly intervals over 14 months. Later on, skin snips were made at three to four-months intervals. The chimpanzee was bled every two to three weeks (from week -12 to 173) and aliquots of sera were stored frozen until use.

\section{Antigen preparation}

The preparation of a soluble $O$. volvulus adult worm extract has been described in detail (LOBOS \& WEISS, 1985). After collagenase digestion of frozen nodules, worms were homogenized, extracted at $4^{\circ} \mathrm{C}$ overnight in $7 \mathrm{mM}$ phosphate buffer ( $\mathrm{pH} 7 \cdot 2$ ) containing $18 \mathrm{mM} \mathrm{NaCl}$. The extract was dialysed against the same buffer and ultracentrifuged $\left(100,000 \mathrm{~g}, 4^{\circ} \mathrm{C}, 2\right.$ hours $)$. The soluble antigen was stored in small aliquots at $-70^{\circ} \mathrm{C}$ until use.

\section{Monoclonal antibodies}

The monoclonal antibodies (Gib 13, Gib 85, Gib 86) directed against the membrane of $O$. gibsoni eggs were prepared at the Immunoparasitology Unit of the Walter \& Eliza Hall Institute of Medical Research, Melbourne. Their preparation has been described in detail (FORSYTH et al., 1984). Monoclonal antibodies from ascites fluid (after ammonium sulphate-precipitation and dialysis) were received at a protein concentration of $4 \mathrm{mg} / \mathrm{ml}$. Gib 13 and Gib 85 were labelled with ${ }^{125}$ I by the Chloramine $T$ method to a specific activity of $0.2 \mu \mathrm{Ci} / \mu \mathrm{g}$ protein.

\section{Enzyme-linked immunosorbent assay (ELISA)}

Polystyrene microtitre plates were coated with the $O$. volvulus antigen (diluted in $60 \mathrm{mM}$ sodium carbonate buffer, $\mathrm{pH} \mathrm{9.6)} \mathrm{at} \mathrm{the} \mathrm{optimal} \mathrm{protein} \mathrm{concentration} \mathrm{of} 2.5 \mu \mathrm{g} / \mathrm{ml}$. All sera $(0.2 \mathrm{ml})$ were tested in parallel at a single dilution of 1:160. A horseradish-peroxidase labelled goat anti-human IgG (heavy and light chain) antiserum (Miles Lab., No. $61-230$ ) and a goat anti-human IgM ( $\mu$ chain specific) 
antiserum (Tago Inc., No. 2392) were used at dilutions of $1: 1500$ and $1: 1000$, respectively. Optical densities (OD) were read at $492 \mathrm{~nm}$ on a Multiscan photometer (Flow Lab.). Wells with readings over 2 were measured at $1: 5$. Mean OD values of three pre-infection sera were deducted from all test results.

\section{Competitive radio-immunoassay (CRIA)}

To detect host antibodies competing with the binding of ${ }^{125}$ I-labelled monoclonal antibodies (Gib 13, Gib 85) for the $O$. volvulus antigen, wells of polyvinyl microtitre plates were sensitized with $100 \mu \mathrm{l}$ of the soluble adult worm antigen (at $2.5 \mu \mathrm{g}$ protein $/ \mathrm{ml}$ ). After post-coating (with $0.5 \%$ bovine serum albumin (BSA) in phosphate buffered saline (PBS) for $30 \mathrm{~min}$ at $37^{\circ} \mathrm{C}$ ) mixtures of $50 \mu \mathrm{l}$ of chimpanzee serum (tested at two-fold dilutions starting from $1: 10$ ) and $50 \mu \mathrm{l}$ of labelled Gib $13(40,000 \mathrm{cpm} /$ well $)$ or Gib $85(84,000 \mathrm{cpm} /$ well) were incubated overnight at room temperature. After three washes with PBS (containing 0.05\% Tween 20) the bound radioactivity was measured in a gamma counter (MR 252, W\&W electronics). The serum dilution which gave a $50 \%$ reduction of binding was determined graphically $(\log / \log$ blot $)$. Total activity bound was $5 \%$ for Gib 13 and $2 \%$ for Gib 85.

\section{Immunoradiometric assay (IRMA) for antigen detection}

Details on the development of these immunoradiometric assays have been given elsewhere (FORSYTH et al., 1984). For this study, we used two combinations of monoclonal antibodies for antigen detection: ${ }^{125} \mathrm{I}-\mathrm{Gib} 13 / \mathrm{Gib} 13$ and ${ }_{125}$ I-Gib 85/Gib 86. The optimized protocol for antigen detection in the chimpanzee serum was the following: wells of polyvinyl microtitre plates were sensitized (overnight at room temperature) with the "catching" antibodies (50 $\mu \mathrm{l}$ Gib 13 or Gib 86) at protein concentrations of $10 \mu \mathrm{g} / \mathrm{ml}$ (in PBS). Before use, they were post-coated with $0.5 \%$ BSA (30 min, $37^{\circ} \mathrm{C}$ ). For detecting circulating antigen, $25 \mu l$ of the test serum (diluted 1:2) was pre-incubated with ${ }^{125}$ I-labelled Gib 13 or Gib $85(60,000 \mathrm{cpm})$ for 16 hours at room temperature in uncoated microtitre plates and then transferred to the corresponding antibody-coated plates. The second incubation was for another 4 hours at $37^{\circ} \mathrm{C}$. Then the plate was washed, cut and counted. Results were expressed as:

\section{Antigen Index:}

Counts per minute (cpm) bound with test serum Mean cpm bound with 3 pre-infection sera

Paper radio-immunosorbent assay (PRIST) for total serum IgE Total serum IgE concentrations were determined using a commercially available paper radio-immunoassay for human IgE (Phadebas-Prist IgE, Pharmacia Diagnostics). 15 selected sera were tested at 1:10 in an IgE-free diluent supplied by the manufacturer.

\section{Results}

The prepatent period of this chimpanzec, cxperimentally infected with a total of $168 \mathrm{O}$. volvulus third-stage larvae, was rather long: no microfilariae ( $m f f$ ) could be found on 12 occasions during the first 110 weeks of infection. At week 124 (i.e., 29 months after the first and 23 months after the last injection of larvae) mff could be detected in skin snips for the first time. Mff-counts remained low (mean less than $2 \mathrm{mff} / \mathrm{snip}$ ) for over $12 \mathrm{months}$ until the end of the observation period (week 173).

The course of the humoral immune response against $O$. volvulus adult antigens was measured by ELISA detecting total antibody levels or IgM-antibodies (Fig. 1). After the injection of 44 larvae, detection of antibodies was possible from week 14 onwards, just before another 35 larvae were inoculated (week 19). From week 34 to 48 no further increase in antibody levels was observed. Antibody levels were maximal between week 64 and 72 , decreased until week 120 and again increased and levelled off at the end of the test period. In comparison, IgM antibodies against $O$. volvulus were only detectable from week 20 onwards (Fig. 1). Their levels were high from week 30 through 72 , but fell, thereafter, to low values.

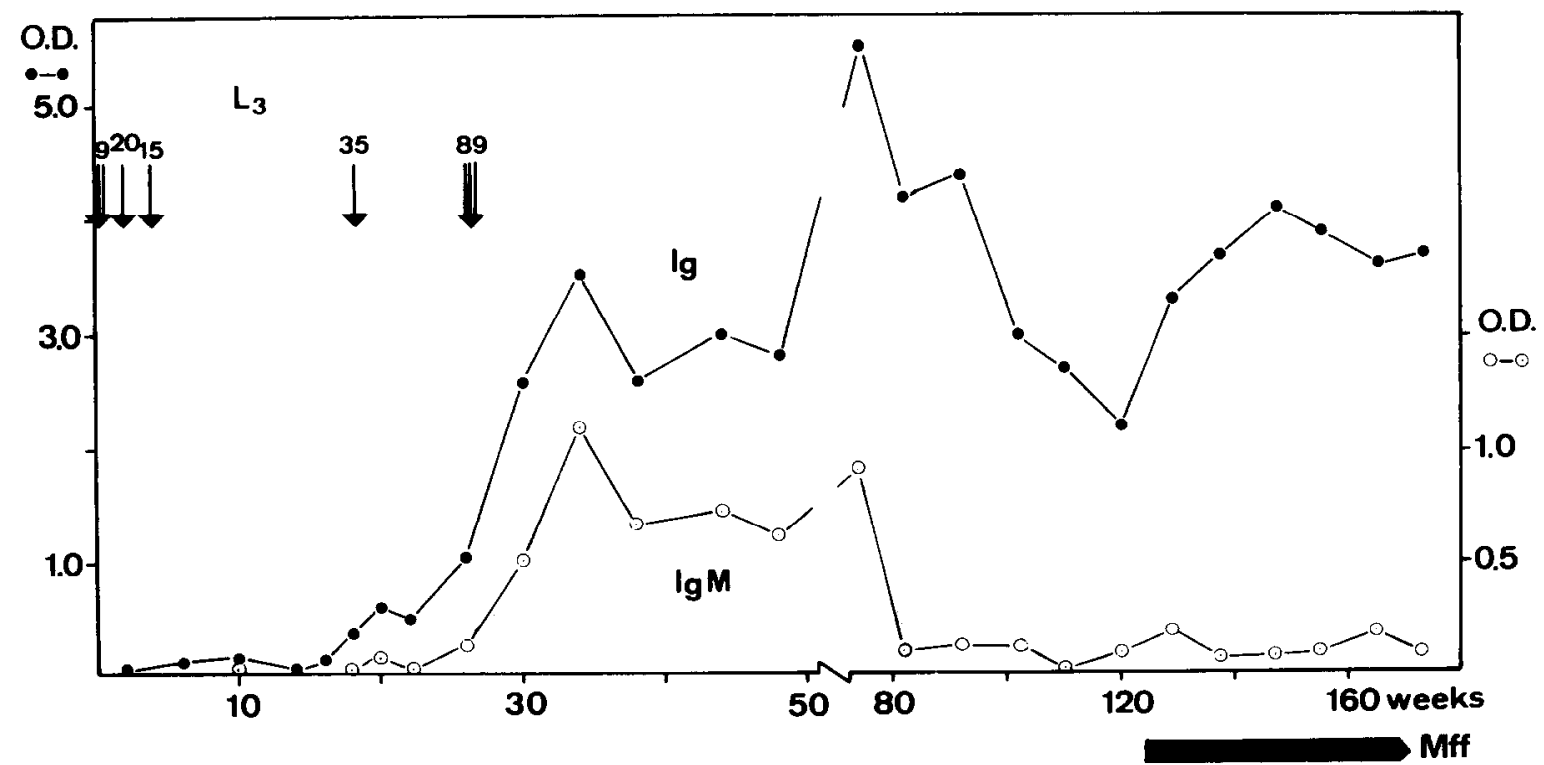

Fig. 1. Serum antibody levels (closed circles: all Ig classes, open circles: IgM) against an adult $O$. volvulus antigen measured by ELISA (OD: optical density at $492 \mathrm{~nm}$ ). Arrows indicate when third stage larvae $\left(\mathrm{L}_{3}\right)$ were inoculated, black bar when microfilariae (Mff) could be detected in skin snips. 
A

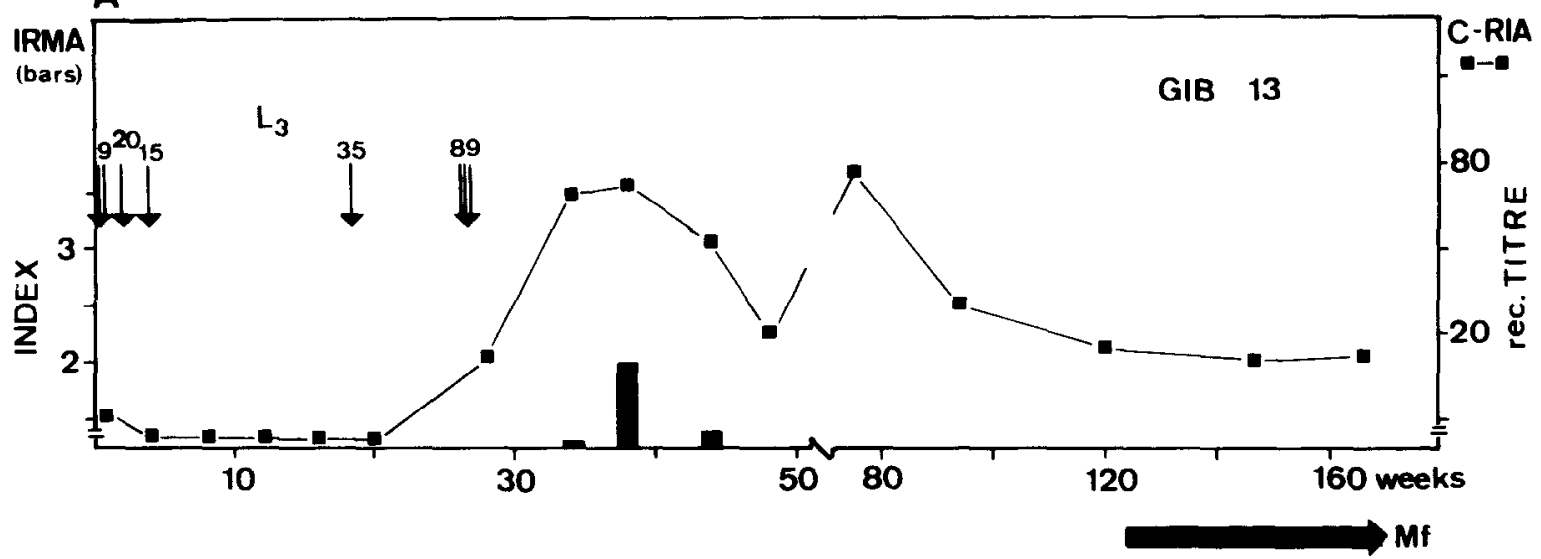

B

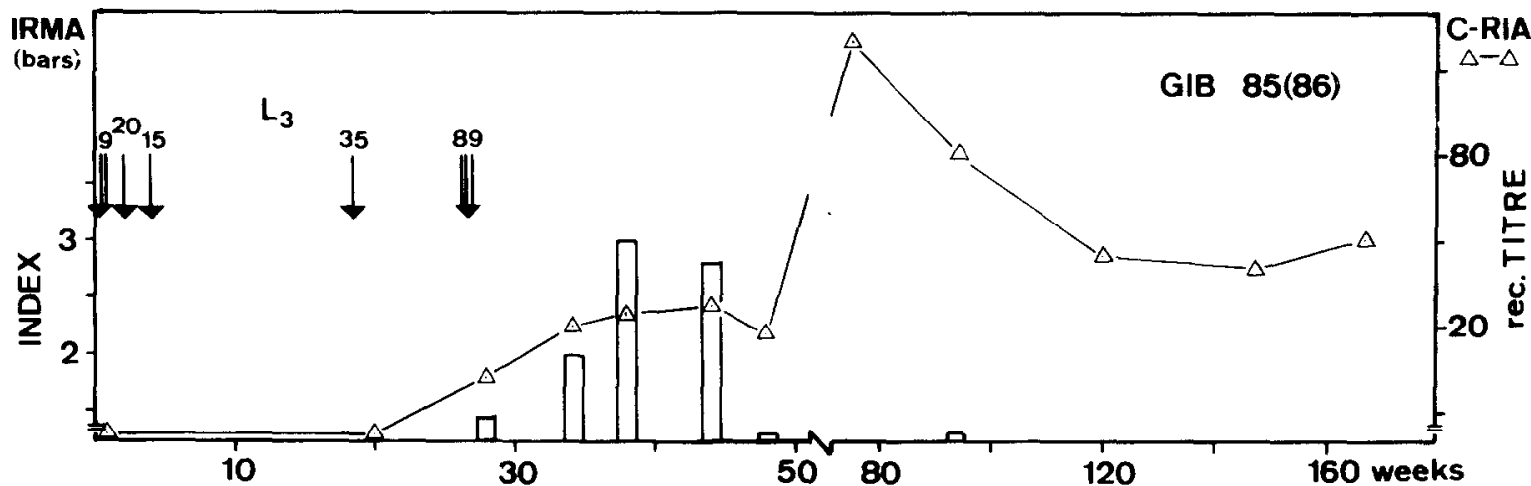

Fig. 2. Detection of circulating antigen by IRMA expressed as antigen index (vertical bars) and serum antibodies (curves) competing with the binding of a monoclonal antibody to $O$. volvulus antigens (gven as reciprocal titres for $50 \%$ inhibition)

A: using ${ }^{125}$ I-Gib $13 /$ Gib 13 for IRMA, and ${ }^{125}$ I-Gib 13 for the competitive radioimmunoassay (C-RIA)

B: using ${ }^{125}$ I-Gib $85 /$ Gib 86 for IRMA and ${ }^{125}$ I-Gib 85 for the C-RIA

No marked increase in the total IgE serum levels could be detected during the course of infection: the highest values $(150,165 \mathrm{kU} / \mathrm{l})$ were detected at weeks 74 and 120 . The total $\mathrm{IgE}$ content of a pre-infection serum was $65 \mathrm{kU} / 1$.

Results of the antigen detection assays are given in Fig. 2. No circulating antigen could be detected by IRMAs using either ${ }^{125}$ I-Gib $13 /$ Gibl3 (Fig. 2A) or ${ }_{125}$ I-Gib $85 /$ Gib 86 (Fig. 2B) in seven sera taken from week 2 through 28 (Antigen Index 1.5). The second combination of monoclonals allowed the detection of parasite antigen in sera taken at weeks 34,38 and 44 , whereas seven sera (after week 44 ) were negative. The Gib 13/13 IRMA was only positive at week 38 .

Host antibodies interfering with the binding of $\mathrm{Gib}$ 13 or Gib 85 to $O$. volvulus antigens were detectable from week 28 onwards. The reciprocal serum titres (reducing the binding of the monoclonal antibody to $50 \%$ ) are given in Fig. 2. For Gib 13, inhibition was highest with sera from week 36 (when antigen detection was possible) and again at week 74 . For Gib 85 , the levels of interfering host antibodies remained low during the time period when antigen could be detected (weeks 34 to 44). Thereafter, a sharp increase in antibody titres was observed. Highest antibody titres (1:200) were measured at week 74
(Fig. 2B). Thereafter, antibody titres fell to 1:40 and levelled off from week 120

\section{Discussion}

The exact time period for the development of $O$. volvulus infective larvae to mature adult worms is unknown. In experimentally infected chimpanzees the prepatent period for a rain-forest "strain" varied between 10 and 26 months (DUKE, 1980). This reflects the fact that the parasitological proof of an infection-by demonstration of skin microfilariaedepends not only on the presence of sexually mature worms but also on the probability of mating and on a sufficient accumulation of microfilariae in the skin. In the chimpanzee studied, no microfilariae could be found in skin snips during the first 26 months of infection. Repeated infections over a period of six months made the correlation of immunological findings and parasitological events difficult. However, the analysis of circulating antigen levels by IRMA may allow the following conclusions: circulating antigens were detectable for a short time before patency, i.e., weeks 34,38 and 44 after infection. The origin of this antigen is likely to be egg debris released from female worms during production and hatching of microfilariae since the monoclonal antibodies Gib 
13, Gib 86 and Gib 85 bind to egg membranes of a number of filarial species including $O$. volvulus (Forsyth \& Weiss, unpublished observations). Measurements of circulating antigens in bovine onchocerciasis have shown that increased levels of the target antigens of the Gib 85/86 IRMA are associated with embryogenesis (FoRSYTH et al., 1984). Treatment of $O$. gibsoni-infected cattle with the microfilaricidal drugs diethylcarbamazine and avermectin did not alter antigen levels. In contrast, drugs that caused disruption of embryogenesis increased specific antigen concentrations in the serum. Assuming a similar relationship between the Gib 85/86 target antigen levels and embryogenesis in human onchocerciasis, the $O$. volvulus-infected chimpanzee was therefore confronted with egg antigens by at least 34 weeks after infection. Indeed egg antigens may have been encountered even earlier, i.e., between weeks 20 and 26. Serum antibodies specific for the target epitopes of Gib 13 and Gib 85 were found by CRIA at this time. From this data one could argue that infective larvae reach maturity six to seven months after infection. This would be in agreement with the observation that the shortest prepatent period for a rain-forest "strain" of $O$. volvulus lies between nine and ten months (DUKE, 1980). The rather late detection of microfilariae in this animal might be either due to the small number of microfilariae produced or to early immune responses controlling microfilariae. The latter hypothesis has recently been discussed when microfilarial densities and serological data from young children living in a hyperendemic area of West Africa had been analysed (KARAM \& WEISS, 1985). In vector-controlled areas it has been shown that some children only become skin snip positive three years after the interruption of transmission (PROST, 1980).

Significant rises in serum antibodies to $O$, volvulus adult worm extract were noted from week 16 onwards, i.e., 10 to 16 weeks after the inoculation of a total of 44 infective larvae but before a booster injection of 35 larvae (Fig. 1). This rise in antibody titres may reflect an immune response to antigens released by juvenile worms which undergo a significant increase in size. From week 22 onwards, a further increase in antibody titres was observed. At this time also IgM antibodies to the parasite became detectable (Fig. 1). Similarly, antibodies reactive with the target epitopes of Gib 13 and Gib 85 were detected at this time (Fig. 2). The rise in antibody titres correlates with some parasitological event such as the onset of embryogenesis predicted by antigenaemic data. Egg-antigen reactive antibodies detected by CRIA were found in all but the first 20 weeks of infection (Fig. 2). The high immunogenicity of these antigens suggest that detection of antibodies to antigens or epitopes specific for $O$. volvulus eggs may be diagnostic for both early and patent stages of $O$. volvulus infections. High titres of antibodies reactive with Gib 85/86 target epitopes have been found in human onchocerciasis patients (FORSYTH, 1984, unpublished thesis).

In later stages of infection, repeated exposures to infective larvae made the interpretation of serological data a matter of speculation. A further increase in antibody titres (weeks 48 to 74 ) might reflect a response to additional adult worms, perhaps derived from the 89 larvae given at week 27 . The decline in antibody titres from week 74 to 120 (Fig. 1)-also found for antibodies reacting with the monoclonal target epitopes on eggs (Fig. 2)-might be due to an increased release of egg materials by a greater number of adult female worms interacting with serum antibodies. A similar explanation could be attributed to the decline of host antibodies against the Gib 13 target epitope between weeks 36 and 48 (Fig. 2). Increasing antibody titres were observed during the patent period, although $\operatorname{IgM}$ antibody levels remained very low (Fig. 1).

Detection of circulating antigens by Gib 13 or Gib 85/86 monoclonal antibodies was only possible for a short time during prepatency: IRMAs were positive at weeks 34,38 and 44 using Gib 85/86 and at week 38 using Gib 13 (Fig. 2). The main factor interfering with antigen detection was host antibodies: antibodies competing for the binding of Gib 13 an Gib 85 to $O$. volvulus worm extract were already detectable from week 28 onwards (Fig. 2). It seems that antigen (or immune complexes) could only be detected when the competing host antibodies were at low concentrations or had low affinities. In a preliminary study on human onchocerciasis, the same phenomenon was observed (FORSYTH, 1984): antigen detection was only possible in $48 \%$ of adult individuals (>15 years) with $O$. volvulus skin microfilariae using Gib 85/86 IRMA and competing host antibodies were detectable in most individuals. In contrast, Gib 13 has successfully been used for antigen detection in lymphatic filariasis (FORSYTH et al. 1985): detection of egg antigens in the serum of infected individuals correlated with the presence and the intensity of both microfilariaemia and disease manifestations. In these patients, however, only low titres of host antibodies to the Gib 13 target epitope could be detected. In a recent study on antigen detection in dogs with heartworm disease, it has been reported that even low concentrations of host antibodies reduced the ability of the IRMA to detect circulating Dirofilaria immitis antigens (HAMILTON \& SCOTT, 1984).

Strategies towards the specific detection of prepatent onchocerciasis using monoclonal antibodies as diagnostic tools have recently been discussed (WEISS, 1985). The amplification effect of the immune system favours the detection of host antibodies. Speciesspecific antibodies could be detected using either purified antigens or $O$. volvulus-specific monoclonal antibodies to developing larvae or juvenile worms. For an optimal antigen detection test one should focus on non-immunogenic parasite products. Sera of experimentally infected primates and longitudinal seroepidemiological surveys in endemic areas are needed to evaluate specific assays with high predictive values to achieve a reliable diagnosis of prepatent onchocerciasis.

\section{Acknowledgements}

We wish to thank Regina Rufener and Wyn Davies for their excellent technical assistance. For the preparation of the Onchocerca antigen we used nodules kindly provided by Drs. Herz, Herzog and Thür (Acha Hospital, Acha-Tugi and Ad Lucem Hospital, Bafang, Cameroon),

$$
\text { References }
$$

Barbiero, V. K. \& Trpis, M. (1984). Transmission of onchocerciasis by local black flies on the Firestone Rubber Plantation, Harbel, Liberia. American fournal of Tropical Medicine and Hygiene, 33, 586-594. 
Duke, B. O. L. (1962). Experimental transmission of Onchocerca volvulus from man to a chimpanzee. Transactions of the Royal Society of Tropical Medicine and Hygiene, $56,271$.

Duke, B. O. L. (1980). Observations on Onchocerca volvulus in experimentally infected chimpanzees. Tropenmedizin und Parasitologie, 31, 41-54.

Forsyth, K. P., Mitchell, G. F. \& Copeman, D. B. (1984). Onchocerca gibsoni: Increase of circulating egg antigen with chemotherapy in bovines. Experimental Parasitology, 58, 41-55.

Forsyth, K. P., Spark, R., Kazura, J., Brown, G. V., Peters, P., Heywood, P., Dissanaike, S. \& Mitchell, G. F. (1985). A monoclonal antibody-based immunoradiometric assay for detection of circulating antigen in Bancroftian filariasis. Foumal of Immunology, 134, 11721177.

Hamilton, R. G. \& Scott, A. L. (1984). Immunoradiometric assay for quantitation of Dirofilaria immitis antigen in dogs with heartworm infections. American foumal of Veterinary Research, 45, 2055-2061.

Karam, M. \& Weiss, N. (1985). Seroepidemiological investigations of onchocerciasis in a hyperendemic area of West Africa. American foumal of Tropical Medicine and Hygiene, 34, 907-917.

Lobos, E. \& Weiss, N. (1985). Immunochemical comparison between worm extracts of Onchocerca volvulus from savanna and rain-forest. Parasite Immunology, 7, 333 . 347.

Prost, A. (1980). Latence parasitaire dans l'onchocercose. Bulletin de l'Organtsation mondiale de la Santé, 58, 923-925.

Weiss, N. (1985). Monoclonal antibodies as an investigative tool in onchocerciasis. Review of Infectious Diseases, 7, 826-830.

Accepted for publication 15th Fune, 1985.

\title{
ROYAL SOCIETY OF TROPICAL MEDICINE AND HYGIENE
}

\author{
Society Award 1986
}

\section{CHALMERS MEDAL}

THE CHALMERS MEDAL is awarded each year in recognition of research contributing to the knowledge of tropical medicine or tropical hygiene. Only persons of 45 years of age or under on the ist June of the year of the award will be eligible.

Fellows are reminded that nomination forms for both awards must be lodged with the Society not later than 31st October 1986.

See p. 25 of the 1985 Yearbook 